\title{
Untargeted Metabolomics for the Diagnosis of Exocrine Pancreatic Insufficiency in Chronic Pancreatitis
}

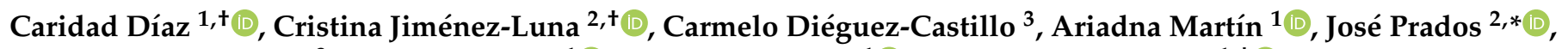 \\ José Luis Martín-Ruíz ${ }^{3}$, Olga Genilloud ${ }^{1}{ }^{1}$, Francisca Vicente ${ }^{1}{ }^{1}$, José Pérez del Palacio ${ }^{1, \neq(\mathbb{D}}$ \\ and Octavio Caba ${ }^{2, \ddagger}$ \\ 1 Fundación MEDINA, Centro de Excelencia en Investigación de Medicamentos Innovadores, \\ 18012 Granada, Spain; caridad.diaz@medinaandalucia.es (C.D.); ariadna.martin@medinaandalucia.es (A.M.); \\ olga.genilloud@medinaandalucia.es (O.G.); francisca.vicente@medinaandalucia.es (F.V.); \\ jose.perezdelpalacio@medinaandalucia.es (J.P.d.P.) \\ 2 Center of Biomedical Research (CIBM), Institute of Biopathology and Regenerative Medicine (IBIMER), \\ University of Granada, 18012 Granada, Spain; crisjilu@ugr.es (C.J.-L.); ocaba@ugr.es (O.C.) \\ 3 Department of Gastroenterology, San Cecilio University Hospital, 18012 Granada, Spain; \\ carmelo89dc@gmail.com (C.D.-C.); jlmartin@ugr.es (J.L.M.-R.) \\ * Correspondence: jcprados@ugr.es; Tel.: +34-958-248-819 \\ $\dagger$ These authors contributed equally to this work. \\ $\ddagger$ These are Co-senior authors.
}

check for updates

Citation: Díaz, C.; Jiménez-Luna, C.; Diéguez-Castillo, C.; Martín, A.; Prados, J.; Martín-Ruíz, J.L.; Genilloud, O.; Vicente, F.; Palacio, J.P.d.; Caba, O. Untargeted Metabolomics for the Diagnosis of Exocrine Pancreatic Insufficiency in Chronic Pancreatitis. Medicina 2021, 57, 876. https://doi.org/10.3390/ medicina57090876

Academic Editor: Rinaldo Pellicano

Received: 28 July 2021

Accepted: 23 August 2021

Published: 26 August 2021

Publisher's Note: MDPI stays neutral with regard to jurisdictional claims in published maps and institutional affiliations.

Copyright: (c) 2021 by the authors. Licensee MDPI, Basel, Switzerland. This article is an open access article distributed under the terms and conditions of the Creative Commons Attribution (CC BY) license (https:/ / creativecommons.org/licenses/by/ $4.0 /)$.
Abstract: Background and Objectives: The clinical manifestations and course of chronic pancreatitis $(\mathrm{CP})$ are often nonspecific and variable, hampering diagnosis of the risk of exocrine pancreatic insufficiency (EPI). Development of new, reproducible, and non-invasive methods to diagnose EPI is therefore a major priority. The objective of this metabolomic study was to identify novel biomarkers associated with EPI. Materials and Methods: We analyzed 53 samples from patients with CP, 32 with and 21 without EPI, using an untargeted metabolomics workflow based on hydrophilic interaction chromatography coupled to high-resolution mass spectrometry. Principal component and partial least squares-discriminant analyses showed significant between-group differentiation, and univariate and multivariate analyses identified potential candidate metabolites that significantly differed between samples from CP patients with EPI and those without EPI. Results: Excellent results were obtained using a six-metabolic panel to diagnose the presence of EPI in CP patients (area under the ROC curve $=0.785$ ). Conclusions: This study confirms the usefulness of metabolomics in this disease setting, allowing the identification of novel biomarkers to differentiate between the presence and absence of EPI in CP patients.

Keywords: metabolomics; exocrine pancreatic insufficiency; chronic pancreatitis; biomarker; diagnosis; high-resolution mass spectrometry

\section{Introduction}

Chronic pancreatitis $(\mathrm{CP})$ is a progressive inflammatory disease characterized by irreversible morphological changes of the pancreatic gland, by fibrosis, and by impairment of exocrine and endocrine functions [1]. Worldwide, CP affects $0.4-5 \%$ of the population, reducing the life quality and expectancy of affected patients [2]. The diagnosis of CP is largely based on the presence of morphological or functional changes, but no standard diagnostic criteria or techniques have been established [3,4]. It is crucial to detect CP before its last stages, when there is a high risk of serious complications (e.g., diabetes mellitus, stenosis, pancreatic cancer, etc.) and the substantial restoration of pancreatic function becomes highly challenging [5].

Exocrine pancreatic insufficiency (EPI) consists of the destruction of the pancreatic parenchyma, with a loss of acinar cells and/or obstruction of pancreatic ducts, leading to 
an inadequate secretion of pancreatic enzymes and bicarbonate to maintain normal digestion [6]. CP is the most frequent cause of EPI in adults [7]. The diagnosis of EPI is currently based on maldigestion-related symptoms, nutritional markers, and noninvasive pancreatic function tests (e.g., fecal elastase-1 [FE-1]) [8]. Direct methods are more sensitive but are laborious, expensive, and invasive [9], whereas indirect methods are less costly, easier to perform, and non-invasive, but are only moderately sensitive for the early diagnosis of EPI $[10,11]$.

The study of metabolism is an emerging and powerful tool for identifying novel biomarkers to achieve early diagnosis and/or evaluation of the course of different diseases [12]. Metabolites represent the end products of gene transcription and protein expression and therefore characterize the phenotype of an organism at a given time [13]. Hence, analysis of differences between normal and altered metabolic pathways may yield new biomarkers for diagnosis [14]. The aim of untargeted metabolomics is to analyze metabolites in an unbiased manner and has recently become widely applied to evaluate metabolic pathway changes associated with alterations [15]. High-resolution mass spectrometry (HRMS) is highly useful in untargeted metabolomics by combining the ability to determine masses within $3 \mathrm{ppm}$ with the technology to investigate unknown compounds by collision-induced dissociation (or MS/MS), allowing exploration of relationships between parent ions and their fragments [16].

The objective of this metabolic study was to use hydrophilic interaction chromatography (HILIC) coupled to HRMS to identify potential biomarkers for the diagnosis of EPI in serum samples from CP individuals; a metabolite-based predictive model was developed to discriminate between patients with and without EPI.

\section{Materials and Methods}

\subsection{Sample Collection}

Our study included 53 samples from CP patients. Baseline characteristics are detailed in Table 1.

Alcoholic habits (defined by the intake of at least one standard drink unit of alcohol per day), sex, and cardiovascular events were evaluated using chi-squared distribution and found to be non-significant $(p=0.1478$ for alcoholic habit, $p=0.659$ for cardiovascular events, and $p=0.0102$ for sex.

In order to achieve maximum reproducibility, the variation in parameters related to patients and sampling (fasting state, time of day of sampling, etc.) was minimized, using blood samples obtained from patients at San Cecilio University Hospital (Granada, Spain) between February and September 2017. The study was approved by the ethics committee of the hospital (approval code: 1269-M1-19), and all clinical investigations were conducted according to the principles of the Declaration of Helsinki. Written informed consent was obtained from all patients before their enrolment in the study.

Clotting of samples was activated by collecting blood from selected patients in BC vacutainer SSTII Advance tubes with silica (Becton Dickinson, Franklin Lakes, NJ, USA), centrifuging for $10 \mathrm{~min}$ at $2450 \mathrm{rpm}$, and aspirating the supernatant, which was stored at $-80^{\circ} \mathrm{C}$.

Table 1. Baseline characteristics of patients with chronic pancreatitis.

\begin{tabular}{lccc}
\hline \multicolumn{1}{c}{ Characteristic } & CP Patients (\%) & EPI (\%) & NO-EPI (\%) \\
\hline Number & 53 & $32(60.4)$ & $21(39.6)$ \\
Age (years) & 55.7 & 56.7 & 54.3 \\
* Sex & & & \\
$\quad$ Male & $44(83)$ & $30(93.7)$ & $14(66.7)$ \\
$\quad$ Female & $9(17)$ & $2(6.3)$ & $7(33.3)$ \\
\hline
\end{tabular}


Table 1. Cont.

\begin{tabular}{|c|c|c|c|}
\hline Characteristic & CP Patients (\%) & EPI (\%) & NO-EPI (\%) \\
\hline \multicolumn{4}{|l|}{ Diagnostic test } \\
\hline Endoscopic ultrasonography & $38(71.7)$ & $21(65.6)$ & $17(81)$ \\
\hline Computed Tomography & $10(18.9)$ & $6(18.7)$ & $4(19)$ \\
\hline Abdominal ultrasound & $3(5.6)$ & $3(9.4)$ & $0(0)$ \\
\hline Anatomopathological study & $2(3.8)$ & $2(6.3)$ & $0(0)$ \\
\hline \multicolumn{4}{|l|}{ * Alcoholic habit } \\
\hline Yes & $34(64.2)$ & $23(71.9)$ & $11(52.4)$ \\
\hline $\mathrm{No}$ & $19(35.8)$ & $9(28.1)$ & $10(47.6)$ \\
\hline \multicolumn{4}{|l|}{ Smoking habit } \\
\hline Yes & $34(64.2)$ & $27(84.4)$ & $7(33.3)$ \\
\hline No & $19(35.8)$ & $5(15.6)$ & $14(66.7)$ \\
\hline \multicolumn{4}{|l|}{ Body Mass Index } \\
\hline Overweight/Obesity (>25) & $24(45.3)$ & $10(31.3)$ & $14(66.7)$ \\
\hline Normal weight $(18>\mathrm{BMI}<25)$ & $28(52.8)$ & $21(65.6)$ & $7(33.3)$ \\
\hline Underweight $(<18)$ & $1(1.9)$ & $1(3.1)$ & $0(0)$ \\
\hline \multicolumn{4}{|l|}{ * Cardiovascular events } \\
\hline Yes & $4(7.6)$ & $2(6.3)$ & $2(9.5)$ \\
\hline No & $49(92.4)$ & $30(93.7)$ & $19(90.5)$ \\
\hline \multicolumn{4}{|l|}{ Abdominal pain } \\
\hline Yes & $24(45.3)$ & $16(50)$ & $8(38.1)$ \\
\hline No & $29(54.7)$ & $16(50)$ & $13(61.9)$ \\
\hline \multicolumn{4}{|l|}{ Diarrhea } \\
\hline Yes & $12(22.6)$ & $10(31.3)$ & $2(9.5)$ \\
\hline No & $41(77.4)$ & $22(68.7)$ & $19(90.5)$ \\
\hline \multicolumn{4}{|l|}{ * Diabetes } \\
\hline Yes & $26(49.1)$ & $20(62.5)$ & $6(28.6)$ \\
\hline No & $27(50.9)$ & $12(37.5)$ & $15(71.4)$ \\
\hline \multicolumn{4}{|l|}{ Complications $^{1}$} \\
\hline Yes & $31(58.5)$ & $19(59.4)$ & $12(57.1)$ \\
\hline No & $22(41.5)$ & $13(40.6)$ & $9(42.9)$ \\
\hline \multicolumn{4}{|l|}{ Hospital readmissions ${ }^{2}$} \\
\hline Yes & $21(39.6)$ & $13(40.6)$ & $8(38.1)$ \\
\hline No & $32(60.4)$ & $19(59.4)$ & $13(61.9)$ \\
\hline \multicolumn{4}{|l|}{ Treatment } \\
\hline Endoscopic & $8(15.1)$ & $6(18.7)$ & $2(9.5)$ \\
\hline Surgical & $13(24.5)$ & $10(31.3)$ & $3(14.3)$ \\
\hline Analgesic & $30(56.6)$ & $21(65.6)$ & $9(42.9)$ \\
\hline Substitutive enzyme & $29(54.7)$ & $22(68.7)$ & $7(33.3)$ \\
\hline Oral antidiabetics & $26(49.1)$ & $20(62.5)$ & $6(28.6)$ \\
\hline Statins/Fibrates & $16(30.2)$ & $11(34.4)$ & $5(23.8)$ \\
\hline
\end{tabular}

$\overline{{ }^{1} \text { Compressive (pseudocyst or abscess), stenotic (biliary o duodenal), or vascular (pseudoaneurysm or splenoportal }}$ thrombosis) ${ }^{2}$ Hospital admissions in a follow-up period of 12 months. ${ }^{*}$ Chi square distribution $p$ value $>0.01$.

\subsection{Diagnosis of Exocrine Pancreatic Insufficiency}

The exocrine pancreatic function of $\mathrm{CP}$ patients was assessed according to FE-1 levels measured with the BioServ Diagnostics Pancreatic Elastase ELISA kit, following the manufacturer's instructions (BioServ Analytics and Medical Devices Ltd., Rostock, Germany) and considering FE-1 $\geq 200 \mu \mathrm{g} / \mathrm{g}$ as normal pancreatic function, FE-1 $=100-200 \mu \mathrm{g} / \mathrm{g}$ as mild-moderate EPI, and FE- $1<100 \mu \mathrm{g} / \mathrm{g}$ as severe EPI.

\subsection{Metabolite Extraction}

AcN (1:8 sample/AcN) was added to serum samples and shaken for 2 min to remove proteins. After centrifugation at $15,200 \mathrm{rpm}$ for $10 \mathrm{~min}$ at $4{ }^{\circ} \mathrm{C}$, supernatants were collected and transferred to vials and then evaporated using the GeneVac HT-8 evaporator (Savant, Holbrook, NY, USA). AcN/water (50:50) with $0.1 \%$ formic acid was used to reconstitute 
obtained dry residues, shaking for $1 \mathrm{~min}$. All samples were kept at $4{ }^{\circ} \mathrm{C}$ throughout analytical procedures.

\subsection{HILIC-HRMS Analysis}

Chromatographic separation was performed as follows: $0.00-0.10 \mathrm{~min} 99 \%$ eluent $B$, 0.10-7.00 min 30\% eluent B, 7.00-7.10 min 99\% eluent $B$, and $7.10-10.00$ min $99 \%$ eluent $B$, using a Waters Xbridge BEH amide column $(2.1 \times 150 \mathrm{~mm}, 2.5 \mu \mathrm{m})$. Column temperature was maintained at $45^{\circ} \mathrm{C}$. The elution flow rate was $400 \mu \mathrm{L} / \mathrm{min}$. Agilent Series 1290 was used as LC instrument.

Mass detection was carried out using an SCIEX Triple TOF 5600 quadrupole-time-offlight mass spectrometer in ESI (-) mode (SCIEX, Concord, ON, Canada) for HILIC analysis. Fragmentation and mass spectra were obtained by operating the TripleTOF 5600 using a TOF method and an information-dependent acquisition (IDA) technique to simultaneously collect full-scan HRMS and MS/MS information.

The IDA technique was used to fragment the eight most intense ions. Exact mass calibration was automatically performed every eight injections. An MP sample was run every 50 samples to identify impurities from the solvents or extraction procedure and to test carryover contamination from intense peaks. A QC sample was injected every 10 chromatographic runs to check variability in the analysis.

\subsection{Data Set Creation}

The retention time (RT) and mass/charge $(\mathrm{m} / \mathrm{z})$ were evaluated to study the analytical reproducibility using PeakView software (version 1.0 with Formula Finder plug-in version 1.0, AB SCIEX, Concord, ON, Canada). Raw data were processed with MarkerView software (version 1.2.1, AB SCIEX, Concord, ON, Canada), which performs peak detection, alignment, and data filtering.

Peak detection was carried out using an algorithm in the RT range of 1-8 min and removing background noise (50 cps). Peak alignment was achieved using RT and $\mathrm{m} / \mathrm{z}$ tolerances of $0.15 \mathrm{~min}$ and $15 \mathrm{ppm}$, respectively. Next, a filter was applied by "presence" to retain masses appearing in at least 5 samples in the study case groups, only considering monoisotopic peaks. Finally, a filtering procedure with $t$-test $(p<0.05)$ and fold-change $(>2)$ was employed to identify differentially expressed mass signals between MP samples and case study samples.

\subsection{Analytical Validation}

Metaboanalyst 3.0 Web Server was used to transform the data matrix into a Gaussiantype distribution by total area sums, auto scaling, and cube transformation. The study was evaluated by the non-supervised PCA method. Variables with unacceptable reproducibility (RSD $>30 \%$ ) were excluded. In the statistical validation, $R^{2}$ and $Q^{2}$ explained the goodness of fit and prediction, respectively. PLS-DA allowed the identification of outliers.

\subsection{Statistical Analysis}

Univariate analysis was performed with the Wilcoxon-test $(p<0.05)$, determining the statistical significance of between-group differences. Multivariate analysis was also conducted, carrying out PCA and a PLS-DA to identify $m / z$ values responsible for the separation between groups. Finally, the fold change $(>1.2)$ between groups was considered as election criterion for variability in the amounts of metabolites.

\subsection{Biomarker Identification}

Selected molecular components were identified by searching in Metlin, Human Metabolome Database, KEGG, and Lipid Maps databases, using a mass tolerance of $<5$ ppm. Next, PeakView software (version 1.0 with Formula Finder plug-in version 1.0, AB SCIEX, Concord, ON, Canada) was used to determine molecular formulas. $m / z$ with the same molecular formula in experimental and database estimations were further ana- 
lyzed by studying their experimental MS/MS spectra with information provided by the MassBank, NIST 2014 MS/MS, Human Metabolome Database, and Metlin databases.

\subsection{Biomarker Evaluation}

The sensitivity and specificity of the proposed biomarkers were tested using the receiver operator characteristic (ROC) curve, a non-parametric measure of biomarker utility. Univariate and multivariate ROC analyses were performed to evaluate the clinical value of the candidates as biomarkers individually and in combination with others.

\section{Results}

EPI was diagnosed or ruled out in CP patients using the FE- 1 test: $60.4 \%$ of patients with CP patients were diagnosed with EPI (FE- $1<200 \mu \mathrm{g} / \mathrm{g}$ ) and the remaining $39.6 \%$ were not (NO-EPI). Among those diagnosed with EPI, 87.5\% had severe EPI (FE- $1<100 \mu \mathrm{g} / \mathrm{g}$ ).

After alignment and filtering processes, a data matrix of 1262 metabolite features was obtained, 302 of which were monoisotopic. Among these, 254 features were differentially expressed in case samples (EPI and NO-EPI) and MP samples. After normalization, six of these variables were discarded due to unacceptable variability (RSD $>30 \%$ ). The PCA score plot for the remaining 248 variables (Figure 1A) revealed a close clustering of the QC samples, indicating that the separation observed between EPI and NO-EPI was mainly due to biological factors, and the PLS-DA score plot (Figure 1B) suggested that it was feasible to discriminate between these patients. Three outliers were detected and excluded from the analysis. The $Q^{2}$ value for the predictive ability of the PLS-DA model to discriminate between EPI and NO-EPI was 0.15 and the $R^{2}$ value was 0.30 , with a good separation between the groups in the PLS-DA model. Out of the total variance, $63.7 \%$ was explained by two components alone (Figure 1B).
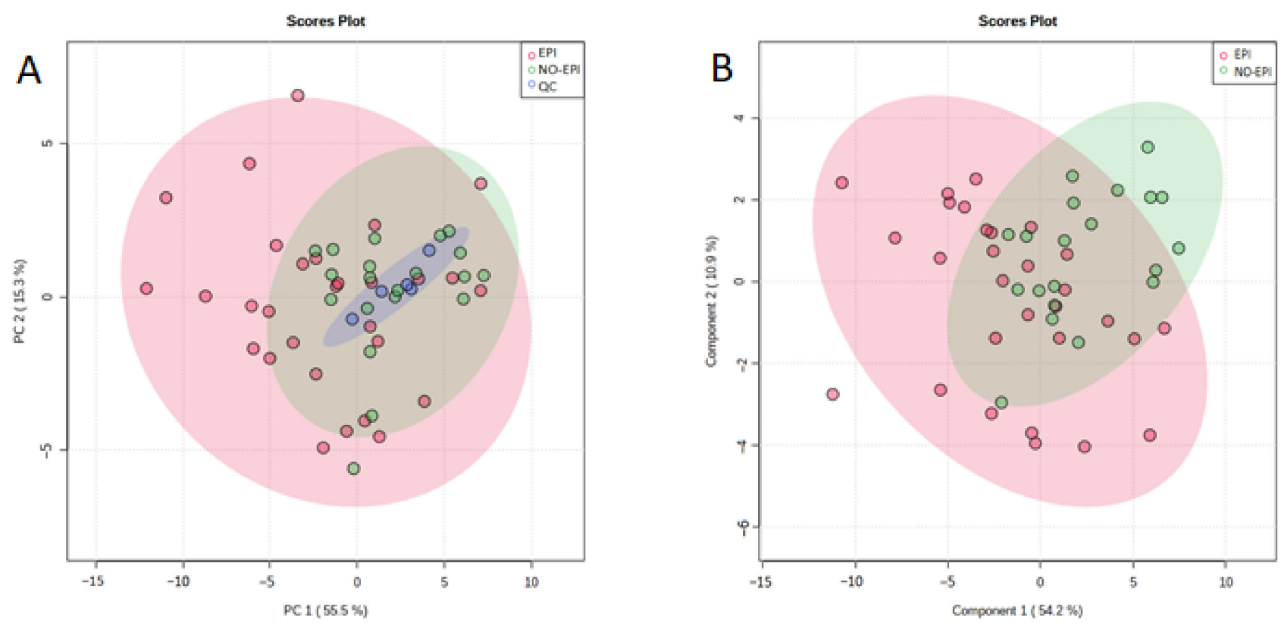

Figure 1. (A) Principal component analysis score plot; (B) partial least squares-discriminant analysis score plot. Pink dots correspond to EPI patients, green dots to NO-EPI patients, and blue dots to quality control samples.

Out of these 248 variables, 12 had a $p$-value $<0.05$. Fold-change calculations (higher than 1.2) showed that 11 metabolites were regulated in opposite directions in patients with versus without EPI. These metabolites were tentatively identified, but only 7 of them could be matched in the database used (Table 2). The remaining significant metabolites could not be assigned an identity $(\mathrm{m} / z 303.9204$ at $3.9 \mathrm{~min}, 841.9492$ at $3.0 \mathrm{~min}, 977.9249$ at $3.1 \mathrm{~min}$ and 1045.915 at $3.2 \mathrm{~min}$ ). In all cases, these molecules were increased in EPI patients. Four phospholipids classified as phosphatidylserines (PS) and one phosphatidylcholine (PC) were increased in the EPI group, together with a peptide formed with arginine, threonine, and proline, although their position is unknown, and pentasine. 
Table 2. Detailed information of the potential biomarkers of exocrine pancreatic insufficiency.

\begin{tabular}{cccccc}
\hline $\boldsymbol{m} / \boldsymbol{z}$ & RT $(\mathbf{m i n})$ & Tentative Identification & $\boldsymbol{\Delta} \mathbf{p p m}$ & Adduct & Fold Change \\
\hline 417.2103 & 1 & Arginine-threonine-proline & 0 & $+\mathrm{F} . \mathrm{A} .-\mathrm{H}$ & 1.74 \\
610.3721 & 1.3 & PC $(16: 0 / 5: 0(\mathrm{OH}))$ & 1 & $-\mathrm{H}$ & 1.43 \\
634.3334 & 2.1 & Pentasine & 1 & $-\mathrm{H}-\mathrm{H}_{2} \mathrm{O}$ & 1.29 \\
828.5726 & 1.3 & PS (R1COOH + R2COOH $=39: 2)$ & 4 & $-\mathrm{H}$ & 1.21 \\
852.5767 & 1.2 & PS (R1COOH + R2COOH $=41: 4)$ & 1 & $\mathrm{H}$ & 1.24 \\
876.5739 & 1.2 & PS (R1COOH + R2COOH = 43:6) & 2 & $\mathrm{H}$ & 1.39 \\
\hline
\end{tabular}

Biomarkers were selected according to $t$-test $(p<0.05)$ and fold change (higher than 1.2) results. RT: Retention time. Fold change expressed as the ratio of the two averages (EPI/NO-EPI).

The potential of the candidate biomarkers was further evaluated by constructing univariate ROC curves to obtain the area under the ROC curve (AUC). Two metabolites showed an AUC $>0.75$ (Figure 2). Two phosphatidylserines were the best individual biomarkers: PS (R1COOH + R2COOH $=41: 4)$, with an AUC of 0.78 (95\% CI 0.63-0.91); and PS (R1COOH + R2COOH = 39:2), with an AUC of 0.75 (95\% CI 0.63-0.91).
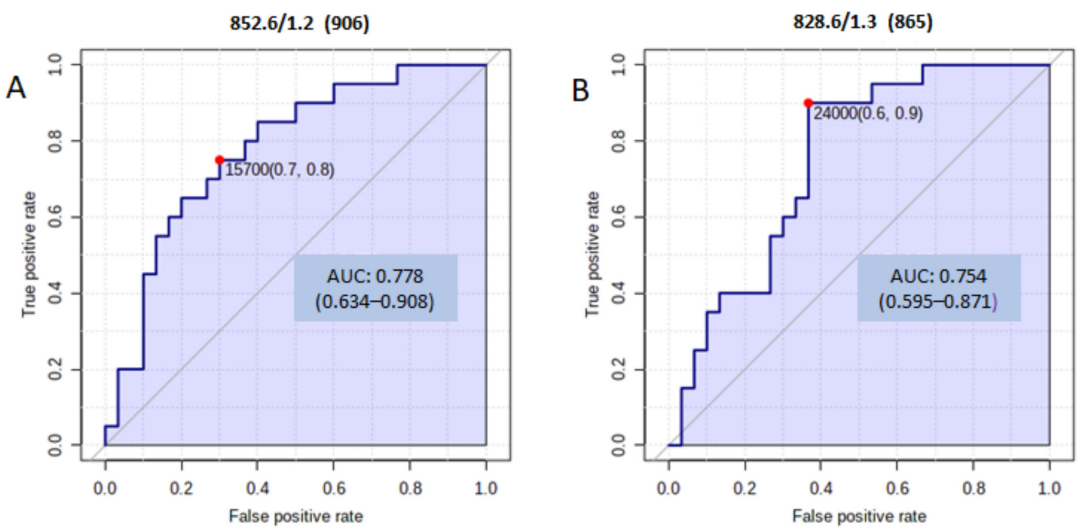

Figure 2. (A) AUC for PS (R1COOH + R2COOH = 41:4); (B) AUC for PS (R1COOH + R2COOH = 39:2).

We applied a random forest model to combine arginine-proline-threonine tripeptide, PC (16:0/5:0(OH)), pentasine, PS ( $\mathrm{R} 1 \mathrm{COOH}+\mathrm{R} 2 \mathrm{COOH}=39: 2)$, PS $(\mathrm{R} 1 \mathrm{COOH}+\mathrm{R} 2 \mathrm{COOH}$ $=41: 4)$, and PS $(\mathrm{R} 1 \mathrm{COOH}+\mathrm{R} 2 \mathrm{COOH}=43: 6$, obtaining an AUC of $0.79(95 \% \mathrm{CI} 0.62-0.92)$ for the combined set (Figure 3A), higher than the AUC for each independent biomarker. The confusion matrix correctly classified 22 patients with pancreatic insufficiency and misclassified 5, and it correctly classified 15 patients without pancreatic insufficient and misclassified 8 (Figure 3B).
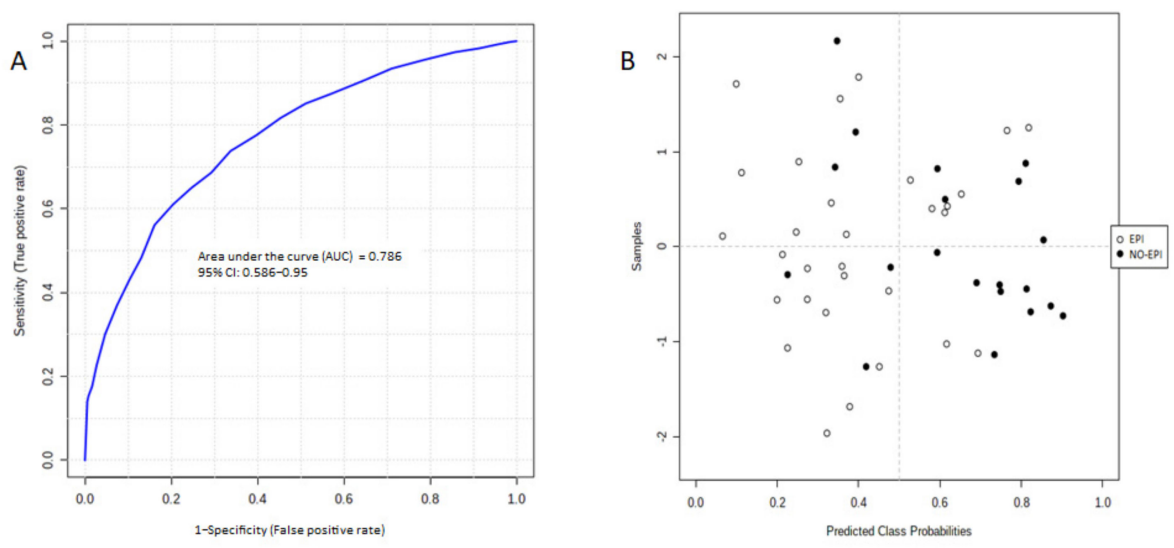

Figure 3. (A) ROC curves for the proposed six-metabolite panel; (B) classification using predicted group probabilities. 


\section{Discussion}

The early diagnosis of EPI in patients with $\mathrm{CP}$ is vital due to the increased risk of malnutrition and cardiovascular events $[17,18]$. Indirect methods are widely used but have a low sensitivity for early stages of EPI [8]. In the present study, a six-metabolite panel proved useful to discriminate between the presence and absence of EPI in patients with CP. One of the first metabolic studies for this purpose used high-pressure liquid-chromatography (LC) to assess the diagnostic capability of the bentiromide test to determine the concentration of p-aminobenzoic acid and its metabolites, and it detected a lower concentration of amines in patients with EPI than in controls. They concluded that this noninvasive method of evaluating pancreatic function could play an important role in the diagnosis of EPI [19].

Among the six metabolites identified as EPI biomarkers in the present study, three of them were phosphatidylserines (PS). These molecules are essential components of eukaryotic cells, present on the inner leaflet of their membranes and with an important role in apoptosis and blood clotting [20]. Interestingly, their levels on the surface of pancreatic cancer cells appear to be higher than on the surface of other types of cancer cell [21], and this abundance has led to proposals for the targeting of PS in combination with gemcitabine in the treatment of pancreatic cancer [22]. In the present study, these molecules were found at higher concentrations in EPI patients, in agreement with previous findings that the PS receptor on pancreatic cells is markedly upregulated in $\mathrm{CP}$, alongside observations implicating pro-apoptotic genes, which are known to play a major role in the destruction underlying EPI [23].

In addition, one phosphatidylcholine (PC) was found at higher concentrations in sera from the EPI group versus NO-EPI group. PC is the major phospholipid component of cell membranes, more commonly found in the outer leaflet, and contributes to proliferative growth and programmed cell death [24]. Zeman et al. analyzed fatty acid (FA) profiles in plasma phosphatidylcholines from $96 \mathrm{CP}$ patients and 108 controls; they found decreased fat intake and intrinsic changes in FA metabolism in the $\mathrm{CP}$ group due to the alteration of delta desaturase activities, resulting in higher monounsaturated FA and lower polyunsaturated FA contents [25].

The highest fold change between studied groups was observed in a peptide formed by arginine, threonine, and proline. Although their position in the peptide is unknown, a search was conducted of a peptide BLAST with all possible tripeptides, finding that arginine-proline-threonine and proline-arginine-threonine are constituent tripeptides of different types of integrin. These proteins have been associated with extracellular matrix destruction processes in inflammatory processes such as pancreatitis [26]. Moreover, it has been demonstrated that the intraperitoneal administration of arginine can induce selective and dose-dependent experimental pancreatitis in rats [27]. The action mechanism of arginine-induced pancreatitis was reported to be mediated by oxidative stress, inflammation, and apoptosis, among other processes [28]. High concentrations of proline have also been related to pancreatic alterations. In 2016, in vitro studies demonstrated that high doses of this cyclic amino acid cause toxicity and beta-cell dysfunction mediated by a decrease in insulin transcription and impairment of mitochondrial oxidative phosphorylation [29].

Finally, our panel also includes pentasine, a carboxylic acid with five carboxyl groups that belongs to the class of organic compounds known as pentacarboxylic acids and derivatives. Several studies have pointed to pentasine as an elastin cross-linking amino acid, which may be related to reduced elastin secretion [30]. Degradation of extracellular matrix proteins such as elastin is considered a prerequisite for the progression of various diseases, including tumor metastases [31]. In addition, extracellular matrices containing elastin-like polypeptide were found to improve islet transplantation outcomes in diabetic mice [32].

The proposed metabolites were identified by using the reported databases, and confirmation of their identity against biological standards would have strengthened the study. A further potential limitation is the lesser representation of females in the study population, attributable to the lower prevalence of $\mathrm{CP}$ among females and in part explained by their 
less frequent exposure to etiological factors [33]. In-depth studies are currently under way to test the capacity of this six-metabolite panel to discriminate between $\mathrm{CP}$ patients with and without EPI in wider samples containing higher percentages of patients with early stage EPI.

\section{Conclusions}

Blood samples from patients with chronic pancreatitis studied with hydrophilic interaction chromatography coupled to mass spectrometry yielded a panel of six metabolites that showed differential expression according to the presence or absence of exocrine pancreatic insufficiency. These results confirm the potential of metabolic studies to improve understanding of the pathogenesis of exocrine pancreatic insufficiency and to identify new diagnostic markers.

\section{Patents}

The patent PCT/EP2020/067255 results from the work reported in this manuscript.

Author Contributions: Conceptualization, J.P.d.P. and O.C.; methodology, C.D.; validation, C.J.-L., C.D. and J.L.M.-R.; formal analysis, A.M.; investigation, C.J.-L.; resources, C.D.-C.; data curation, C.D.; writing - original draft preparation, C.J.-L. and C.D.; writing—review and editing, J.P. and J.P.d.P.; supervision, O.G. and J.P.; project administration, F.V.; funding acquisition, O.C. All authors have read and agreed to the published version of the manuscript.

Funding: This research was funded by the JUNTA DE ANDALUCIA, grants number PC-0498-2017 and PC-0549-2017, and the INSTITUTO DE SALUD CARLOS III, grant number DTS17/00081.

Institutional Review Board Statement: The study was conducted according to the guidelines of the Declaration of Helsinki, and approved by the Ethics Committee of Granada (protocol code: 0893-N-20 on 9 April 2020).

Informed Consent Statement: Informed consent was obtained from all subjects involved in the study.

Data Availability Statement: The data of the study are present at the Fundacion MEDINA. The data will be available upon reasonable request.

Conflicts of Interest: The authors declare no conflict of interest.

\section{References}

1. Kleeff, J.; Whitcomb, D.C. Chronic pancreatitis. Nat. Rev. Dis. Primers 2017, 3, 17060. [CrossRef] [PubMed]

2. Gupte, A.; Goede, D. Chronic pancreatitis. BMJ 2018, 361, k2126. [CrossRef] [PubMed]

3. Dominguez-Munoz, J.E.; Drewes, A.M. Recommendations from the United European Gastroenterology evidence-based guidelines for the diagnosis and therapy of chronic pancreatitis. Pancreatology 2018, 18, 847-854. [CrossRef]

4. Duggan, S.N.; Ní Chonchubhair, H.M. Chronic pancreatitis: A diagnostic dilemma. World J. Gastroenterol. 2016, 22, 2304-2313. [CrossRef]

5. Ramsey, M.L.; Conwell, D.L. Complications of Chronic Pancreatitis. Dig. Dis. Sci. 2017, 62, 1745-1750. [CrossRef] [PubMed]

6. Hart, P.A.; Conwell, D.L. Challenges and Updates in the Management of Exocrine Pancreatic Insufficiency. Pancreas 2016, 45, 1-4. [CrossRef]

7. de la Iglesia-Garcia, D; Vallejo-Senra, N. Increased Risk of Mortality Associated with Pancreatic Exocrine Insufficiency in Patients with Chronic Pancreatitis. J. Clin. Gastroenterol. 2018, 52, e63-e72. [CrossRef] [PubMed]

8. Dominguez-Muñoz, J.E. Diagnosis and treatment of pancreatic exocrine insufficiency. Curr. Opin. Gastroenterol. 2018, 34, 349-354. [CrossRef] [PubMed]

9. Anaizi, A.; Hart, P.A. Diagnosing Chronic Pancreatitis. Dig. Dis. Sci. 2017, 62, 1713-1720. [CrossRef]

10. González-Sánchez, V.; Amrani, R. Diagnosis of exocrine pancreatic insufficiency in chronic pancreatitis: 13C-Mixed Triglyceride Breath Test versus Fecal Elastase. Pancreatology 2017, 17, 580-585. [CrossRef]

11. Vanga, R.R.; Tansel, A. Diagnostic Performance of Measurement of Fecal Elastase-1 in Detection of Exocrine Pancreatic Insufficiency: Systematic Review and Meta-analysis. Clin. Gastroenterol. Hepatol. 2018, 16, 1220-1228. [CrossRef] [PubMed]

12. Madji Hounoum, B.; Blasco, H. Liquid Chromatography-High-Resolution Mass Spectrometry-Based Cell Metabolomics: Experimental Design, Recommendations, and Applications. Trends Analyt. Chem. 2016, 75, 118-128. [CrossRef]

13. Guo, H.; Guo, H.; Zhang, L.; Tang, Z.; Yu, X.; Wu, J.; Zeng, F. Metabolome and Transcriptome Association Analysis Reveals Dynamic Regulation of Purine Metabolism and Flavonoid Synthesis in Transdifferentiation during Somatic Embryogenesis in Cotton. Int. J. Mol. Sci. 2019, 20, E2070. [CrossRef] 
14. Zhang, A.; Sun, H. Metabolomics for Biomarker Discovery: Moving to the Clinic. BioMed Res. Int. 2015, 2015, 354671. [CrossRef] [PubMed]

15. Cajka, T.; Fiehn, O. Toward Merging Untargeted and Targeted Methods in Mass Spectrometry-Based Metabolomics and Lipidomics. Anal. Chem. 2016, 88, 524-545. [CrossRef]

16. Ríos Peces, S.; Díaz Navarro, C. Untargeted LC-HRMS-Based Metabolomics for Searching New Biomarkers of Pancreatic Ductal Adenocarcinoma: A Pilot Study. SLAS Discov. 2017, 22, 348-359. [CrossRef] [PubMed]

17. Min, M.; Patel, B. Exocrine Pancreatic Insufficiency and Malnutrition in Chronic Pancreatitis: Identification, Treatment, and Consequences. Pancreas 2018, 47, 1015-1018. [CrossRef]

18. de la Iglesia, D.; Vallejo-Senra, N. Pancreatic exocrine insufficiency and cardiovascular risk in patients with chronic pancreatitis: A prospective, longitudinal cohort study. J. Gastroenterol. Hepatol. 2019, 34, 277-283. [CrossRef] [PubMed]

19. Durie, P.R.; Yung-Jato, L.Y. Bentiromide test using liquid-chromatographic measurement of p-aminobenzoic acid and its metabolites for diagnosing pancreatic insufficiency in childhood. J. Pediatr. 1992, 121, 413-416. [CrossRef]

20. Kay, J.G.; Fairn, G.D. Distribution, dynamics and functional roles of phosphatidylserine within the cell. Cell Commun. Signal. 2019, 17, 126. [CrossRef]

21. Sharma, B.; Kanwar, S.S. Phosphatidylserine: A cancer cell targeting biomarker. Semin. Cancer Biol. 2018, 52, 17-25. [CrossRef]

22. Köninger, J.; Balaz, P. Phosphatidylserine receptor in chronic pancreatitis: Evidence for a macrophage independent role. Ann. Surg. 2005, 241, 144-151. [CrossRef] [PubMed]

23. N'Guessan, K.F.; Davis, H.W.; Chu, Z.; Vallabhapurapu, S.D.; Lewis, C.S.; Franco, R.S.; Olowokure, O.; Ahmad, S.A.; Yeh, J.J.; Bogdanov, V.Y.; et al. Enhanced Efficacy of Combination of Gemcitabine and Phosphatidylserine-Targeted Nanovesicles against Pancreatic Cancer. Mol. Ther. 2020, 28, 1876-1886. [CrossRef]

24. Ridgway, N.D. The role of phosphatidylcholine and choline metabolites to cell proliferation and survival. Crit. Rev. Biochem. Mol. Biol. 2013, 48, 20-38. [CrossRef] [PubMed]

25. Zeman, M.; Macášek, J.; Burda, M.; Tvrzická, E.; Vecka, M.; Krechler, T.; Staňková, B.; Hrabák, P., Jr.; Žák, A. Chronic pancreatitis and the composition of plasma phosphatidylcholine fatty acids. Prostaglandins Leukot. Essent. Fatty Acids 2016, 108 , 38-44. [CrossRef]

26. Osman, M.O.; Jensen, S.L. Acute pancreatitis: The pathophysiological role of cytokines and integrins. New trends for treatment? Dig. Surg. 1999, 16, 347-362. [CrossRef]

27. Hegyi, P.; Rakonczay, Z., Jr.; Sári, R.; Góg, C.; Lonovics, J.; Takács, T.; Czakó, L. L-arginine-induced experimental pancreatitis. World J. Gastroenterol. 2004, 10, 2003-2009. [CrossRef] [PubMed]

28. Abdelzaher, W.Y.; Ahmed, S.M.; Welson, N.N.; Marraiki, N.; Batiha, G.E.; Kamel, M.Y. Vinpocetine ameliorates L-arginine induced acute pancreatitis via Sirt1/Nrf2/TNF pathway and inhibition of oxidative stress, inflammation, and apoptosis. Biomed. Pharmacother. 2021, 133, 110976. [CrossRef] [PubMed]

29. Liu, Z.; Jeppesen, P.B.; Gregersen, S.; Bach Larsen, L.; Hermansen, K. Chronic Exposure to Proline Causes Aminoacidotoxicity and Impaired Beta-Cell Function: Studies In Vitro. Rev. Diabet. Stud. 2016, 13, 66-78. [CrossRef]

30. Akagawa, M.; Suyama, K. Mechanism of formation of elastin crosslinks. Connect. Tissue Res. 2000, 41, 131-141. [CrossRef] [PubMed]

31. Verma, S.; Kesh, K.; Ganguly, N.; Jana, S.; Swarnakar, S. Matrix metalloproteinases and gastrointestinal cancers: Impacts of dietary antioxidants. World J. Biol. Chem. 2014, 5, 355-376. [CrossRef] [PubMed]

32. Lee, K.M.; Kim, J.H.; Choi, E.S.; Kim, E.; Choi, S.K.; Jeon, W.B. RGD-containing elastin-like polypeptide improves islet transplantation outcomes in diabetic mice. Acta Biomater. 2019, 94, 351-360. [CrossRef] [PubMed]

33. Drake, M.; Dodwad, S.J.; Davis, J.; Kao, L.S.; Cao, Y.; Ko, T.C. Sex-Related Differences of Acute and Chronic Pancreatitis in Adults. J. Clin. Med. 2021, 10, 300. [CrossRef] [PubMed] 